\title{
Spontaneous Direction-Changing or Reversing Positional Nystagmus without Changing Head Position during Head-Roll/Head-Hanging Maneuvers: Biphasic Positional Nystagmus
}

\author{
Sertac Yetiser \\ Department of Otorhinolaryngology, Head and Neck Surgery, Anadolu Medical Center, Kocaeli, Turkey
}

\begin{abstract}
Background and Objectives: Conflicting mechanisms have been reported about spontaneous reversal of positional nystagmus during head-roll maneuver in patients with benign paroxysmal positional vertigo (BPPV). The objective of this study is to review the reports about the characteristics and possible mechanisms of reversing positional nystagmus and to present seven new cases. Subjects and Methods: Seven cases (5 males, 2 females; 4 left-sided, 3 right-sided) were recruited among 732 patients with BPPV seen outpatient clinic between 2009 and 2019. Diagnosis of lateral canal canalolithiasis was confirmed when transient geotropic nystagmus was documented during head-roll test. Reversing positional nystagmus was analyzed in each case and clinical characteristics of the patients were documented. Results: The age of patients was ranging between 30 to 64 years $(46.44 \pm 10.91)$. Duration of symptoms was short (21.34 \pm 19.74$)$. Six of them had a story of head trauma. Initial latency was short. First, intense geotropic nystagmus was observed following provocative head-roll position on the affected side. There was short "silent phase". Then, a longer second-phase of reversed nystagmus was noted. Total duration of nystagmus was $78.40 \pm 6.82$ seconds. Maximal slow phase velocity was $24.05 \pm 6.34 \mathrm{deg} / \mathrm{sec}$. All patients were cured with barbeque maneuver. Conclusions: Ipsilateral reversing positional nystagmus during head-roll maneuver is due to lateral canal canalolithiasis. Mechanism is likely to be due to endolymphatic double flow. Bilateral cases may be due to simultaneous co-existence of canalolithiasis and cupulolithiasis. Longer recording of nystagmus is recommended not to miss the cases with spontaneous direction-changing positional nystagmus.

J Audiol Otol 2021;25(1):43-48
\end{abstract}

KEY WORDS: Benign paroxysmal positional vertigo · Positional nystagmus · Head-roll.

\section{Introduction}

Benign paroxysmal positional vertigo (BPPV) is a common peripheral cause of vertigo at outpatient Otolaryngology clinic clinics. Otoconia inside the semicircular canals make the ampullary hair cells sensitive to gravitational forces in patients with BPPV when they turn their head to either side or when they bend their head forward or backward [1]. Posterior canal $\mathrm{BPPV}$ is the most common form which is presented with up

This is an Open Access article distributed under the terms of the Creative Commons Attribution Non-Commercial License (https://creativecommons.org/licenses/by-nc/4.0/) which permits unrestricted non-commercial use, distribution, and reproduction in any medium, provided the original work is properly cited. beating clockwise or counterclockwise torsional nystagmus during Dix-Hallpike maneuver. Lateral canal BPPV is determined during supine head-roll maneuver according to the direction and the severity of nystagmus which is more severe on the affected side in patients with geotropic nystagmus and more severe on the unaffected side in patients with apogeotropic nystagmus. However, presence of latency, fatigability and limited duration of the nystagmus are the main points of peripheral type of positional nystagmus. Clearance of the semicircular canal with liberatory or re-positioning maneuvers provides complete relief of symptoms.

Direction-changing nystagmus without changing the head position can be observed in patients with central pathology 
$[2,3]$. There is no sense of spinning during positional tests in these patients and they do not have any quick relief of balance problem. However, spontaneous changing of direction of the original positional nystagmus is also an interesting variety of BPPV $[4,5]$. A secondary reverse nystagmus in the opposite direction is seen following expiration of the original positional nystagmus after bringing the head to the provoking position. There is no agreement on pathological mechanism and this issue is the subject of discussion. The objective of this study is to review the reports about the characteristics of reversing positional nystagmus and the possible mechanisms. The data of our series with seven patients were also presented.

\section{Subjects and Methods}

Videonystagmographic (VNG) (Micromed, Inc., Chatam, IL, USA) recordings of 732 patients with BPPV seen outpatient clinic between 2009 and 2019 were reviewed. Seven patients ( 5 males and 2 females) had lateral canal BPPV with ipsilateral reversing positional nystagmus (4 left-sided, 3 rightsided). Spontaneous nystagmus was investigated in all patients before starting the test. VNG recording of all patients were reviewed for a possibility of central involvement (gaze-paretic, rebound nystagmus, visual suppression deficit, impairment of smooth pursuit and saccadic eye movements etc.). Patients had normal otoscopic view and normal hearing threshold. Patients with muscular, ocular and neurological symptoms and those having any medication one week prior to test were excluded. Patients with direction changing positional nystagmus were subjected to magnetic resonance imaging of the temporal bone and the brain which were normal. All patients were tested while the patients' eyes were open and covered with wireless infrared camera. Diagnosis of lateral canal canalolithiasis was confirmed when transient geotropic nystagmus was documented during head-roll test which was performed by placing the patient's head rapidly to the right and later to the left while he is in the supine position. The patients were kept in the same position (head to the left or head to the right) for 5 minutes. The test was performed without visual fixation. Verbal informed consent was obtained from each patient. The procedures were in accordance with the ethical standards of the declaration of Helsinki and of institutional review board of the Anadolu Medical Center. Table 1 shows detail analysis of previous nine studies in chronological order [4-12].

\section{Results}

Age of seven patients was ranging between 30 to 64 years $(46.44 \pm 10.91)$. Average duration of symptoms was short
(21.34 \pm 19.74 seconds). Presence of latency, short duration, and adaptation of transient induced nystagmus during headroll maneuver confirmed BPPV. All patients with lateral canal BPPV had geotropic type nystagmus and six of them had a story of minor head trauma. Table 2 shows clinical data of the presented cases. Latency (time between head movement and the appearance of nystagmus) was short (average: $3.32 \pm$ 1.52 seconds). First, an intense geotropic nystagmus (average: $18.02 \pm 3.72$ seconds) was observed following provocative head-roll position on the affected side. Nystagmus frequency slowly declined and then expired. There was a short "silent phase" (average: $8.64 \pm 3.22$ seconds) without nystagmus. Then, a longer second-phase of reversed nystagmus was noted (average: $48.32 \pm 3.78$ seconds). The average total duration of nystagmus was $78.40 \pm 6.82$ seconds. The average maximal slow phase velocity was $24.05 \pm 6.34 \mathrm{deg} / \mathrm{sec}$. Five patients had complete relief of symptoms within 5-7 days after therapeutic maneuvers. Two patients needed repetition of maneuver. None of the patients had any recurrence in 2 years. Schematic view of reversing positional nystagmus in a patient with right-sided geotropic type lateral canal BPPV is illustrated at Fig. 1, 2.

\section{Discussion}

Bàràny was one of the first to report the patients with positional nystagmus characterized by limited duration and fatigability [13]. Interestingly, he claimed an otoconial dysfunction. BPPV is well known since Dix and Hallpike's work in 1952 [13]. It may sometimes appear in atypical forms which do not fit to the classical description. Spontaneous direction changing positioning nystagmus during head-roll maneuver was first reported in 1965 by Stahle and Terins [5]. However, they did not present any underlying pathological mechanism. Pagnini, et al. [6] reported six cases with geotropic type lateral canal BPPV in a series of 15 cases in which they all had spontaneous reversal of nystagmus on the pathological side. They hypothesized that primary ampullopetal endolymphatic flow is followed by ampullofugal flow due to movement of clots in the opposite direction leading to second-phase ageotropic nystagmus [6]. Therefore, endolymphatic reflux and doublephase endolymphatic flow theory was first outlined by Pagnini, et al. [6] in 1989.

In the following reports, Baloh, et al. [7] in 1993 and Nuti, et al. [8] in 1996 have emphasized the adaptation process to interpret the mechanism for the first time. Probably the reason for a new proposal was the observation of new bilateral cases since it was difficult to explain these cases with a "endolymphatic re-flow theory" due to canalolithiasis. De la Meil- 


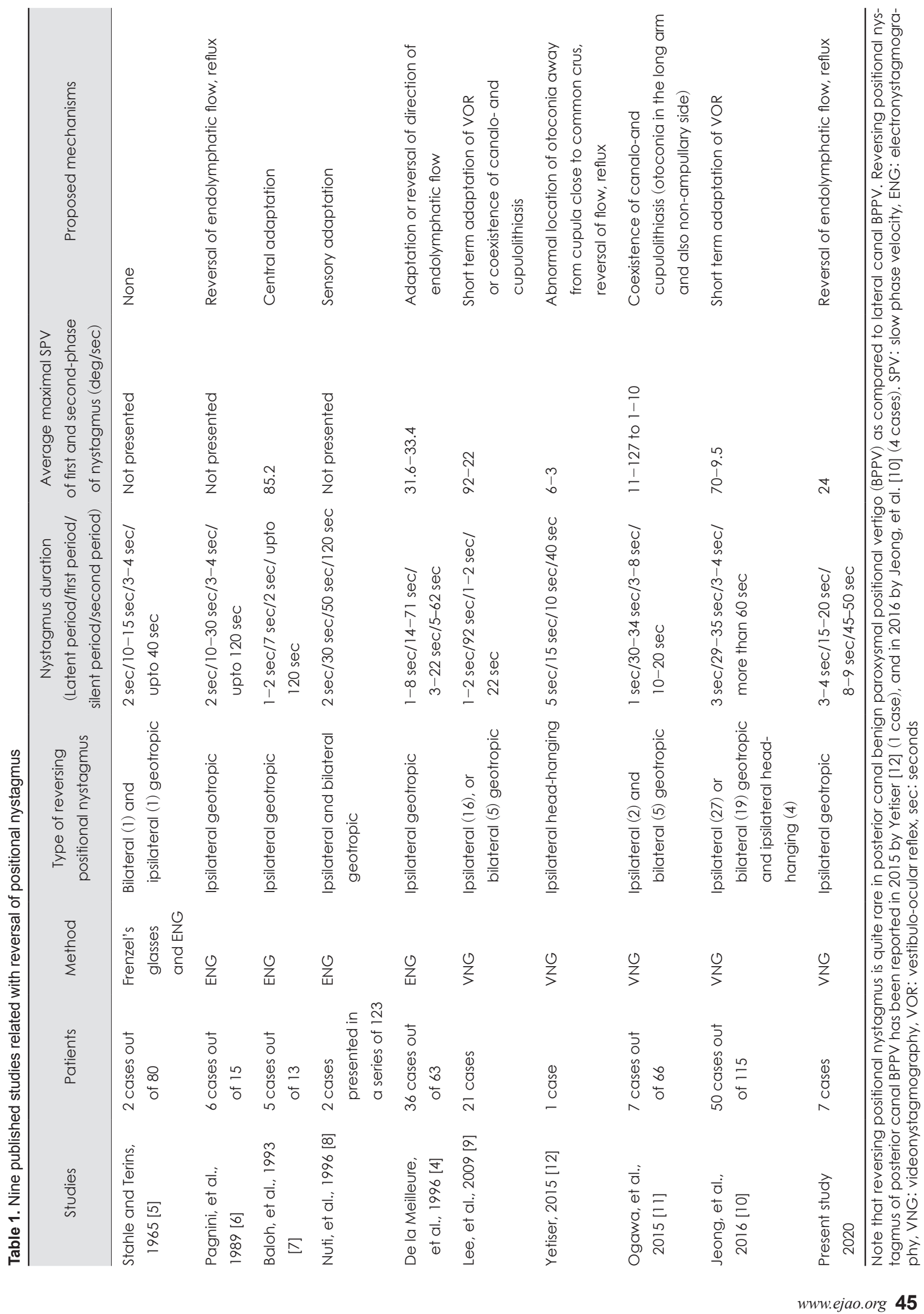


Table 2. Clinical characteristics of patients with reversing positional nystagmus

\begin{tabular}{|c|c|c|c|c|c|}
\hline $\begin{array}{l}\text { Patients' } \\
\text { age and gender }\end{array}$ & Type of BPPV & Side & $\begin{array}{l}\text { Duration of symptoms } \\
\text { (days) }\end{array}$ & Ethiology & $\begin{array}{l}\text { Number of } \\
\text { CRM session }\end{array}$ \\
\hline $30, M$ & Geotropic type LC canalolithiasis & RE & 25 & Tooth extraction & 1 \\
\hline $50, M$ & Geotropic type LC canalolithiasis & RE & 6 & Fall & 1 \\
\hline $64, M$ & Geotropic type LC canalolithiasis & LE & 60 & Idiopathic & 3 \\
\hline $44, M$ & Geotropic type LC canalolithiasis & RE & 7 & Traffic accident & 2 \\
\hline $52, M$ & Geotropic type LC canalolithiasis & LE & 3 & Head trauma & 1 \\
\hline $48, F$ & Geotropic type LC canalolithiasis & RE & 12 & Traffic accident & 1 \\
\hline $37, F$ & Geotropic type LC canalolithiasis & LE & 10 & Bike accident & 1 \\
\hline
\end{tabular}

Note that symptom duration is generally short and majority of patients had a story of trauma. Five patients had relief of symptoms after single barbeque maneuver. However, two patients required multiple maneuvers. BPPV: benign paroxysmal positional vertigo, CRM: canalith re-positioning maneuver, M: male, F: female, LC: lateral canal, RE: right ear, LE: left ear

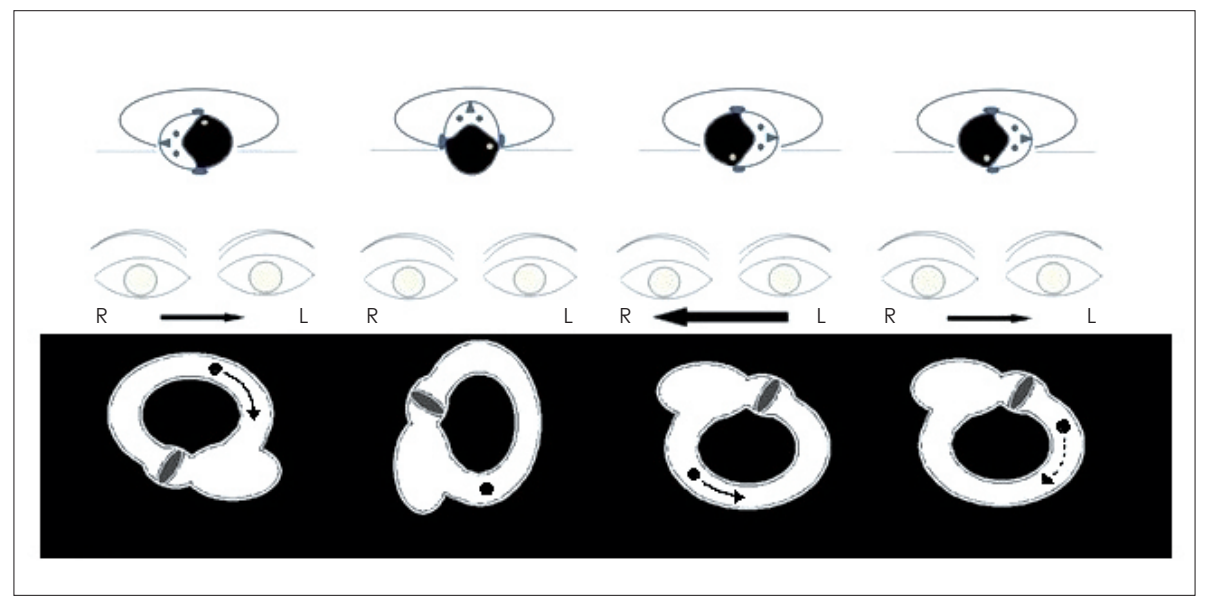

Fig. 1. Schematic view of reversing positional nystagmus in a patient with right-sided geotropic type lateral canal BPPV. In the first position of head-roll maneuver (first picture on the left), a weaker geotropic nystagmus appears when the patient lies on his left side (beating to the left). This is because of ampullafugal endolymphatic flow (away from cupula) of the debris located in the lateral canal. No nystagmus is seen in the central/neutral position. When the patient abruptly turns his head to his right side, a stronger geotropic nystagmus beating to his right is seen. Then, endolymphatic reflux in the opposite direction will pull the debris away from the cupula leading to ampullofugal ageotropic inhibitory nystagmus (last picture). This could be explained by the mass effect of debris itself. Arrows indicate beating direction of the nystagmus. R: right, L: left.

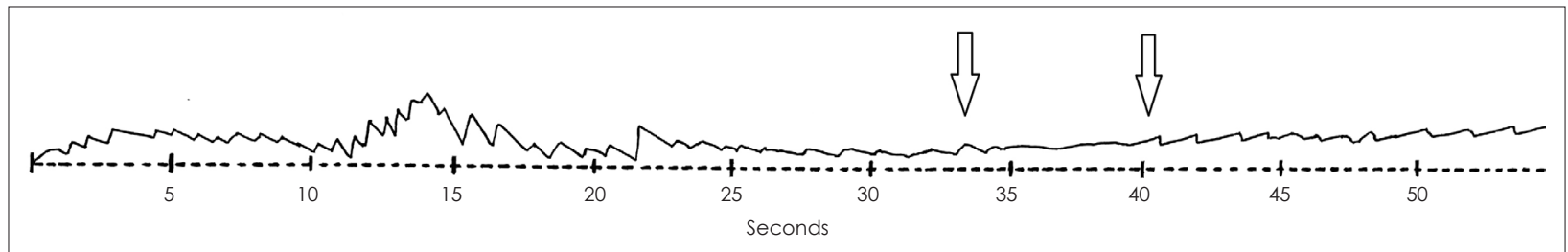

Fig. 2. Reversing positional nystagmus. Note that latency is very short. A right-beating initial nystagmus lasts 35 seconds. After a brief silent period for 7-8 seconds, a less intense nystagmus revers to the left lasting up to 70 seconds (arrows: the end of first and the beginning of second-phase nystagmus).

leure, et al. [4] have reported 36 cases of geotropic type lateral canal BPPV with reversal of the positional nystagmus in which all of them were on the pathological side. However, proposed pathological mechanisms were both adaptation and reversal of direction of endolymphatic flow [4]. Lee, et al. [9] have reported 16 ipsilateral and five bilateral cases. They have proposed short-term sensory adaptation of the vestibuloocular reflex and the possibility of simultaneous co-existence of cupulolithiasis and canalolithiasis for the first time [9]. One of the largest series was presented by Jeong, et al. [10] They have reported 19 bilateral and 27 unilateral cases. Four patients had posterior canal BPPV [10].

Bilateral occurrence of reversing nystagmus during head roll maneuver in patients with lateral canal BPPV is the difficult one to explain. Adaptation mechanism may not be enough to clarify the reversal of nystagmus during head turning to 
the healthy side since increase of perilymphatic potassium concentration would not occur with inhibition of ampullary nerve by the head turning to the healthy side. Besides, it has been reported in bilateral cases that reversed nystagmus seen during head turning to healthy side is usually less intense $[8,9]$. Ogawa, et al. [11] reported seven cases (five bilateral and two ipsilateral) with reversal of nystagmus and proposed the possibility of coexistence of canalolithiasis and cupulolithiasis to explain the pathological mechanism for bilateral and unilateral cases. They have indicated that the debris circulating in the long arm of pathological horizontal canal and at the same time, debris entering the non-ampullary side and sinking to the cupula will cause bilateral or unilateral occurrence of reversing nystagmus. The assumption of the possibility of presence of otoconial debris at more than one different location in the same ear clarifies bilateral cases.

"Second-phase of nystagmus" is an interesting and an important phenomenon. First-phase nystagmus while bringing the head to provocative position is due to gravitational force which is instantaneous, fast, and strong. This leads an excitatory ampullopedal endolymphatic movement toward cupula. Second-phase nystagmus is longer and less severe. This is probably because of impulsive effect of moving debris in the opposite direction due to flow of endolymphatic reflux which results in ampullofugal apogeotropic inhibitory nystagmus. From clinical point of view, second-phase nystagmus is a supporting sign of two important issues. First, it is surely a case of canalolithiasis and tells us that otoconia is freely floating. Reversing of positional nystagmus has not been reported in patients with ageotropic lateral canal BPPV so far. Second, the side with reversing nystagmus associated with geotropic positional nystagmus clearly indicates the side of pathology. All patients in this series benefit from barbeque maneuver in the opposite direction of the side with reversing nystagmus.

The true incidence of this phenomenon is not known. Longer recording time up to 5 minutes has been recommended in patients with geotropic type lateral canal BPPV to catch more cases [10]. However, the underlying cause is also not clear. In the presented series and cases in the previous reports, trauma is the leading factor in majority of them. Maybe, the amount and dispersal of otoconial debris is different than regular cases. It is also interesting to note that slow phase velocity of initial nystagmus in these cases is high (between 30-50 deg/ $\mathrm{sec}$ ) indicating a powerful endolymphatic flow [7]. Reversal of nystagmus in patients with posterior canal BPPV has been reported to be quite rare. The reason is not clear. However, endolymphatic reflux theory would also explain the occurrence of reversal of nystagmus in these cases, if we could assume that the otoconia is located away from the ampulla, close to the common crus $[12,14]$.

Patients with unilateral vestibular dysfunction like vestibular migraine, Meniere disease, vestibular neuronitis may present positional nystagmus. This condition can be regarded as intensification of spontaneous nystagmus by positional maneuvers which is sometimes not quite clear during primary gaze position. Acute unilateral peripheral vestibular dysfunction creates vestibular asymmetry which results from a unilateral change of the resting neural input. In unilateral hypofunction, head movement to each side exposes nonlinearity as specified by Ewald's second law. This type of nystagmus keeps beating as long as the patient's head is held in this position. It is associated with prolonged stimulation of normal vestibulo-oculomotor system [15]. However, peripheral type positional nystagmus in BPPV is confirmed with the presence of latency, short duration, and adaptation of the transient nystagmus.

In conclusion, statements explaining the mechanism of BPPV variants are pretty much theoretical and are based on observational reports. The design of this study is a retrospective review of BPPV variants associated with reversal of positional nystagmus with a presentation of our clinical data. The amount and dispersal of debris or the position of the head and cupula during testing may be related with the occurrence of second-phase nystagmus. Longer recordings of nystagmus in patients with lateral canal involvement is particularly recommended not to miss the cases with directionchanging positional BPPV. From the presented cases and cases in previous reports, we suggest that reversal of positional nystagmus is related with freely floating otoconia in the semicircular canal. Bilateral cases are likely to be simultaneous presentation of co-existence of canalo-and cupulolithiasis in the same ear. This is also important from therapeutical aspects since more maneuvers may be required. Trauma seems to be the main cause. Having a quite intense initial nystagmus in almost all cases, we may consider an excessive amount of otoconial debris entering the canal which create a powerful flow.

\section{Acknowledgments \\ None}

\section{Conflicts of interest}

The author has no financial conflicts of interest.

\section{ORCID iDs}

Sertac Yetiser

https://orcid.org/0000-0003-1430-1026

\section{REFERENCES}

1) Bhattacharyya N, Gubbels SP, Schwartz SR, Edlow JA, El-Kashlan $\mathrm{H}$, Fife $\mathrm{T}$, et al. Clinical practice guideline: benign paroxysmal positional vertigo (Update) executive summary. Otolaryngol Head Neck 
Surg 2017;156:403-16

2) Sakata E, Ohtsu K, Itoh Y. Positional nystagmus of benign paroxysmal type (BPPN) due to cerebellar vermis lesions. Pseudo-BPPN. Acta Otolaryngol Suppl 1991;481:254-7.

3) Dunniway HM, Welling DB. Intracranial tumors mimicking benign paroxysmal positional vertigo. Otolaryngol Head Neck Surg 1998;118:429-36.

4) De la Meilleure G, Dehaene I, Depondt M, Damman W, Crevits L, Vanhooren G. Benign paroxysmal positional vertigo of the horizontal canal. J Neurol Neurosurg Psychiatry 1996;60:68-71.

5) Stahle J, Terins J. Paroxysmal positional nystagmus: an electronystagmographic and clinical study. Ann Otol Rhinol Laryngol 1965;74: 69-83.

6) Pagnini P, Nuti D, Vannucchi P. Benign paroxysmal vertigo of the horizontal canal. ORL J Otorhinolaryngol Relat Spec 1989;51:161-70.

7) Baloh RW, Jacobson K, Honrubia V. Horizontal semicircular canal variant of benign positional vertigo. Neurology 1993;43:2542-9.

8) Nuti D, Vannucchi P, Pagnini P. Benign paroxysmal positional vertigo of the horizontal canal: a form of canalolithiasis with variable clinical features. J Vestib Res 1996;6:173-84.

9) Lee SH, Kim MK, Cho KH, Kim JS. Reversal of initial positioning nystagmus in benign paroxysmal positional vertigo involving the horizontal canal. Ann N Y Acad Sci 2009;1164:406-8.

10) Jeong KH, Shin JE, Shin DH, Kim CH. Direction-reversing nystagmus in horizontal and posterior semicircular canal canalolithiasis. Otol Neurotol 2016;37:767-71.

11) Ogawa $Y$, Ichimura A, Otsuka K, Hagiwara A, Inagaki T, Shimizu S, et al. Spontaneous inversion of nystagmus without a positional change in the horizontal canal variant of benign paroxysmal positional vertigo. J Vestib Res 2015;25:169-75.

12) Yetiser $S$. A new variant of posterior canal benign paroxysmal positional vertigo: a nonampullary or common crus canalolithiasis. Case Rep Otolaryngol 2015;2015:816081.

13) Bárány E. [Diagnosis of symptoms in the area of the otolith enapparates]. Acta Otolaryngol 1920;2:434-7.

14) Yetiser S. Simultaneous and spontaneous reversal of positional nystagmus; an unusual peripheral sign of benign paroxysmal positional vertigo. Otolaryngology Case Reports 2017;3:4-6.

15) Kim CH, Shin JE, Yoo MH, Park HJ. Direction-changing and direction-fixed positional nystagmus in patients with vestibular neuritis and meniere disease. Clin Exp Otorhinolaryngol 2019;12:255-60. 